To appear in AJ

\title{
HST Observations and Models of The Gravitational Lens System MG $0414+0534^{1}$
}

\author{
Emilio E. Falco, Joseph Lehár, and Irwin I. Shapiro \\ Harvard-Smithsonian Center for Astrophysics, 60 Garden St., Cambridge, MA 02138
}

\begin{abstract}
Quadruple gravitational lens systems offer the possibility of measuring time delays for image pairs, microlensing effects, and extinction in distant galaxies. Observations of these systems may be used to obtain estimates of $\mathrm{H}_{0}$ and to study the various mass components of lens galaxies at high redshifts. With the Hubble Space Telescope, we have observed the reddest known gravitational lens system, MG $0414+0534$. We used WFPC2/PC1 to obtain deep, high-resolution images with two filters, F675W and F814W. We present a detailed analysis of all of the components of MG0414, as well as macrolens models. Our main results are: (1) confirmation that MG0414 is inescapably a gravitational lens system; (2) discovery of a blue arc connecting the 3 brightest images of the QSO central core; (3) accurate positions and apparent brightnesses for all 4 known images of the QSO central core and for the lens galaxy G; (4) a good representation of the brightness distribution of $\mathrm{G}$ by elliptical isophotes with a De Vaucouleurs profile, characteristic of an elliptical galaxy; (5) models that consist of simple elliptical potentials and account qualitatively, but not quantitatively, for the HST image positions, arc morphology and radio flux ratios for the images of the QSO central core; (6) a possible new test to distinguish between reddening in the host galaxy of the QSO and in the lens galaxy, based on future accurate measurements of spatial variations in the color of the arc; and (7) the suggestion that microlensing is a plausible cause for the differences between the radio and optical flux ratios for the brightest images, A1 and A2. Further observations and measurements such as of the redshift of the lens galaxy, can be used fruitfully to study microlensing for this system.
\end{abstract}

\footnotetext{
${ }^{1}$ Based on observations made with the NASA/ESA Hubble Space telescope, obtained at the Space Telescope Science Institute, which is operated by AURA under NASA contract NAS5-26555
} 


\section{Introduction}

Among the most spectacular examples of gravitational lensing by isolated galaxies are the quadruple systems of images, with characteristic sizes of a few arcseconds.

MG 0414 + 0534 (hereafter MG 0414) was discovered by the MG radio survey (see, e.g., Hewitt et al. 1989), because of its distinctive "quad" radio morphology (Hewitt et al. 1992, hereafter HTLSB92). The image configuration is the "classic" bright, close double (A1 and A2 separated by 0."4) assumed to straddle a critical curve, plus two additional, fainter images (B and $\mathrm{C}$, each $\sim 2^{\prime \prime}$ from A1 and A2 and from each other). The redshift of each image is $z=2.639 \pm 0.002$ (Lawrence et al. 1995a, hereafter LEJT95; see also Katz \& Hewitt 1993, hereafter KH93; and Lawrence 1996). The lens appears to be a single elliptical galaxy labeled G (Schechter \& Moore 1993, hereafter SM93). Its redshift remains undetermined; attempts with the Keck I telescope initially appeared successful, but later were shown to be a misidentification of Fe II lines at the redshift of the QSO (Lawrence et al. 1995b) as Na D lines at $z \sim 0.5$. The QSO images are exceedingly red, and extinction in the lens galaxy is suspected of being significant (LEJT95).

To further study MG0414, we undertook a series of observations with the Hubble Space Telescope (HST). We describe our observations in $\S 2$, as well as our reduction and analysis of the "raw" HST images. In $\S 3$, we give our astrometry and photometry results for the components of the system. We present a detailed analysis of the properties of the images, with models that account for most of their observed properties in $\S 4$. Finally, our conclusions follow in $\S 5$.

\section{Observations}

We used WFPC2 to obtain 9 deep, high-resolution images with two broad-band filters, F675W and F814W, which are similar, respectively, to Johnson $R$ and $I$. We obtained these data on 8 November 1994, all in fine lock mode. We used filter F675W for 4 of the exposures, one of $1800 \mathrm{sec}$ and 3 of $2100 \mathrm{sec}$. We used filter F814W for the other 5 equal-duration exposures of $2100 \mathrm{sec}$. The MG 0414 system was located near the center of PC1; unsaturated stellar objects on PC1 were aligned from exposure to exposure with a resultant rms scatter of 0.13 pixels (each pixel is $\simeq 0^{\prime \prime} .0455$ ). We combined the exposures for each filter into a single image, to reject most of the numerous cosmic ray "hits" in our exposures. We used standard IRAF" tasks, which eliminated the majority of the "hot"

\footnotetext{
${ }^{1}$ IRAF (Image Reduction and Analysis Facility) is distributed by the National Optical Astronomy Observatories, which are operated by the Association of Universities for Research in Astronomy, Inc., under
} 
pixels in the combined exposures. The combined images are the means of 4 and 5 images for F675W and F814W, respectively, each scaled by its exposure time. We will refer to these combined images as "R" and "I" images, respectively.

We detected the MG0414 system in each band, and found the components that were known from previous ground-based observations (HTLSB92; SM93; Angonin-Willaime et al. 1994, hereafter AVHM94) and from HST observations (Falco 1993). Figures 1 and 2 respectively show $\sim 5$ ".8 and $\sim 23^{\prime \prime} .3$ fields containing MG0414 extracted from the combined " $R$ " images. The figures reveal the expected configuration of 4 point-like images of the core, A1, A2, B and C, as well as the lens galaxy G (Figure 1 labels these components). In addition, our data reveal an arc that we interpret (see §3) as lensed emission from the vicinity of the QSO. We detected a group of objects within $\sim 1^{\prime \prime}$ of one another, $\sim 4^{\prime \prime}$ to the SW of the lens system. Each of the objects in this group is faint and extended (see Figure 2). Hence, we assume it is a compact group of galaxies. Among the other objects in Figure 2 are 3 stars and other faint galaxies.

\section{Astrometry and Photometry}

The components of MG0414 are easily discerned in Figures 2 and 4. Figures 1 and 3 show close-up views of the $R$ and $I$ images, respectively. The apparent "fuzz" surrounding the images of the core in the latter figure is the low-level structure of the PC1 point-spread function (PSF). Although the images of the core are separated by only $\sim 2^{\prime \prime}$, the lensing galaxy was detected by Schechter \& Moore (SM93), under excellent observing conditions $(\mathrm{FWHM}=0$ ".80), and then confirmed by Falco et al. with WFPC1 (Falco 1993), and by Angonin-Willaime et al. (AVHM94). As the figures show, we, too, detected the lens galaxy with WFPC2. From our data, we are able to determine the spatial distribution of the lens galaxy's light emission at high significance levels. We also see clearly object X (SM93) near our detection limit, especially in $R$. Furthermore, one can easily see in both $R$ and $I$ (Figures 1 and 2) arc-like extended emission connecting A2 and B, and joining A1 and A2. The arc is unresolved in the direction from the center of brightness of G. Such an arc can be explained as lensed emission from a single, compact source near the QSO core, as we discuss in $\S 4$.

We used IRAF to measure the pixel coordinates of the centers of brightness and the aperture magnitudes of objects with peak apparent magnitudes of $22.7 \mathrm{mag} / \mathrm{arcsec}^{2}(24.2$ $\left.\mathrm{mag} / \operatorname{arcsec}^{2}\right)$ above the sky level of the $I(R)$ image. The absolute coordinates, e.g., of B,

contract with the National Science Foundation. 
agree with those of Katz \& Hewitt (KH93) at the 0'.08 level, consistent with their absolute positional uncertainty of $0^{\prime \prime} 1$. The images of the core and the galaxy were well above our detection thresholds above the sky level in each of $R$ and $I$. Object $\mathrm{X}$ was higher than the threshold only in $I$, although it is visible by eye in $R$. We estimated its $R$ magnitude by fixing its position to that found in $I$, and comparing its brightness with that of $\mathrm{C}$, whose $R$ and $I$ magnitudes were well determined.

We converted the instrumental magnitudes to Johnson $R$ and $I$ with the method of Holtzman et al. (1996). We used their calibrated zero-points as appropriate for PC1 and for each filter, and the corrections necessary for our nominal CCD gain. We set the magnitude scale for each band using color corrections derived from the ground-based colors of the images of the core and of $\mathrm{G}$. For the images of the core, the mean is $R-I \approx 1.8$; for $\mathrm{G}, R-I \approx 1.6$, both based on AVHM94. Our magnitude estimates follow from the instrumental magnitudes within circular apertures 5 pixels in radius $(\sim 0$ ' 23$)$, except for that of $\mathrm{G}$, which is clearly not point-like (see below), and required a radius of 20 pixels. Tables 1 and 2 show the coordinates and magnitudes of the images of the core, the lens galaxy G, object $\mathrm{X}$, the 3 brightest stars, and the faint, compact group of galaxies $\sim 4^{\prime \prime}$ $\mathrm{SW}$ of $\mathrm{MG} 0414$. We used $\mathrm{C}$ as the origin of coordinates, because it is more isolated than $\mathrm{A} 1, \mathrm{~A} 2$ or $\mathrm{B}$, and suffers less contamination from the arc. The differences between the $R$ and $I$ coordinate offsets of the images of the core are $\leq 0^{\prime \prime} .015$. The maximum errors for centroiding the images of the core, as determined with IRAF, were 0.'02; we adopted these values as the standard errors for their coordinate offsets in the tables. There is also very good agreement between our estimates for the latter offsets, and the analogous estimates of KH93: the two sets differ by $\leq 0^{\prime \prime} 045$, the size of a PC1 pixel.

Our magnitude estimates and those in AVHM94 for A1+A2 (unresolved in their observations), $\mathrm{B}, \mathrm{C}$ and $\mathrm{X}$ differ by $<3 \sigma$, using their errors, which are larger than ours by factors of $\sim 5-10$. Thus, our measurements should represent a substantial refinement of the estimates of the colors of the images of the core and of $\mathrm{X}$ that were discussed in AVHM94. Our magnitude estimates for G are fainter than those in AVHM94 by $\sim 0.7$ and $\sim 0.5 \mathrm{mag}$ in $R$ and $I$, respectively. These represent $\sim 5 \sigma$ deviations, probably indicative of the (lower) accuracy that can be achieved by subtraction of the images of the core in ground-based images of MG0414. Table 3 shows the flux ratios A1/A2, (A1+A2)/B and $\mathrm{C} / \mathrm{B}$ for both of our pass bands, $R$ and $I$. Our ratios are consistent with those of SM93, comparing their $I^{\prime}$ band with our $I$, and also with those of AVHM94 in both $R$ and $I$.

We estimated the apparent brightness of the longer part of the arc with a polygonal aperture containing its emission, save for a PSF avoidance zone 10 pixels in radius from the centers of brightness of the images of the core, which excluded any significant light 
in the PSF wings. We could not obtain a useful estimate of the apparent brightness of the shorter portion of the arc between $\mathrm{A} 1$ and $\mathrm{A} 2$, because the separation $\mathrm{A} 1-\mathrm{A} 2$ is too small to yield magnitudes for the arc without severe contamination. We used IRAF to lay out a polygonal aperture along a $\sim 1^{\prime \prime}$ length of the arc between images $\mathrm{B}$ and A2, thus minimizing contamination by $\mathrm{A} 1, \mathrm{~A} 2$ and $\mathrm{B}$. We calculated $R$ and $I$ brightnesses with the same magnitude zero-points as for the images of the core, but avoided adding the color corrections, because we did not have ground-based estimates. We found $R_{\text {arc }}=24.3 \pm 0.3$, $I_{\text {arc }}=23.0 \pm 0.2$ and, hence, $R_{\text {arc }}-I_{\text {arc }}=1.3 \pm 0.3 \mathrm{mag}$ and $R_{\text {core }}-I_{\text {core }}=2.2 \pm 0.1$ mag; hence the arc is significantly $(2 \sigma$ level) bluer than the QSO core. We also subdivided our aperture into 4 equal, independent, adjacent rectangular apertures, to estimate color variation along the arc. We found no variation, within the standard error of 0.3 mag in our measurements.

We attempted to improve the resolution of our combined images by deconvolution of the PSF in each band, to explore qualitative features of the images. We calculated PSFs appropriate to each band and to PC1 with Tiny Tim v. 4.0 (Krist 1996). Figure 5 shows the results of MEM deconvolution with these PSFs. The deconvolved brightness levels are shown as a contour plot for the $R$ image, where the arc stands out most clearly. This figure shows that the images of the core were sharpened to point-like sources. The longer segment of the arc joining B and A1 remains faint, but the shorter segment joining A1 and A2 can now be seen very clearly. A very small, faint extension also appears to emanate from image C, toward its North, similar to that seen in EVN VLBI observations (Patnaik \& Porcas 1996; henceforth PP96).

We fitted elliptical isophotes to the brightness distribution of $\mathrm{G}$ in $I$, where it is most clearly defined in our combined images. We adjusted the pixel coordinates of the centers of the isophotes, as well as their ellipticities $e_{i}=1-b_{i} / a_{i}$, where $a_{i}$ and $b_{i}$ are the major and minor semi-axes of the $i^{\text {th }}$ isophote, respectively, and the position angles of the $a_{i}$. The coordinate offsets of the center of brightness of $\mathrm{G}$ from $\mathrm{C}$ are listed in Tables 1 and 2. The standard errors in these quantities are $\sim 00^{\prime \prime} 05$, as estimated from the isophote fitting. The mean ellipticity of the fitted isophotes is $e_{G}=0.20 \pm 0.02$, and their mean position angle (E of $\mathrm{N}$ ) on the sky is $\Phi_{G}=71^{\circ} \pm 5^{\circ}$. The main contributor to the standard errors for these elliptical isophotes is the faintness of the outer regions of $\mathrm{G}$. The residuals from the subtraction of the elliptical isophote model from the image were consistent with sky noise, as expected for a good fit.

We also fitted profiles to the distribution of surface brightness of $G$ as a function of the mean semi-major axis of its elliptical isophotes. We convolved the model profiles with the azimuthally-averaged profile of the PSF that we created with Tiny Tim v. 4.0, 
as above, for the $I$ data. Because we used only an azimuthal average of the PSF, the procedure is not significantly sensitive to the details of the PSF wings. We found a good fit $\left(\chi^{2} / D O F \sim 1.7\right.$, where $D O F=38$ is the number of degrees of freedom for the fit), for an " $r^{1 / 4 "}$ De Vaucouleurs law $\left(I=I_{e} \times \exp \left(-7.67\left[\left(r / r_{e}\right)^{1 / 4}-1\right]\right)\right)$ with parameters $r_{e}=1^{\prime \prime} .5 \pm 0^{\prime \prime} .7$ and $I_{e}=24.9 \pm 0.4 \mathrm{mag} / \operatorname{arcsec}^{2}$. Figure 6 is a comparison of our profile data with our DeVaucouleurs profile. We found a better fit $\left(\chi^{2} / D O F \sim 0.8\right)$ for a Hubble-law profile $\left(I=I_{0} /\left(1+\left(r / r_{0}\right)^{2}\right)\right)$, and a worse fit $\left(\chi^{2} / D O F \sim 2.7\right)$ for an isothermal profile $\left(I=I_{0} /\left(1+\left(r / r_{0}\right)^{2}\right)^{1 / 2}\right)$. These results are similar to those obtained by van Dokkum \& Franx (1996) for E galaxies observed with HST in the cluster of galaxies CL 0024 + 016, at $z=0.4$. Such a similarity is a clue that $\mathrm{G}$ may be an elliptical galaxy at $z \sim 0.4$.

\section{Gravitational lens models}

As an application of the astrometry and photometry of $\S 3$, we attempted to estimate parameters of lens models that accounted for the following observed properties of MG0414: (1) the relative coordinates $x_{i}$ and $y_{i}$ of the images of the core and of the center of brightness of $\mathrm{G}(i=\mathrm{A} 1, \mathrm{~A} 2, \mathrm{~B}, \mathrm{C}$ and $\mathrm{G}) ;(2)$ the ratios of core image fluxes $F_{i} / F_{j}$, with $i, j=\mathrm{A} 1$, $\mathrm{A} 2, \mathrm{~B}$ and $\mathrm{C}$ for $i<j$; and (3) the shape and location of the arc.

It is likely that stars in $\mathrm{G}$ do not affect the radio flux ratios by microlensing (Witt, Mao \& Schechter 1995, hereafter WMS95). As in other lensed systems (e.g., 2237+0305, see Falco et al. 1996, and references therein), the optical flux ratios are probably affected by microlensing. Indeed, the optical and radio flux ratios are quite different (e.g., KH93): for example, the ratio A1/A2 is $\sim 2.0$ in $I$ (see Table 3), and $\sim 1.1$ at $8 \mathrm{GHz}$. We attempted to account for the extended emission of the arc pixel-by-pixel, but we arrived at the conclusion that it is too faint to contribute useful constraints based on our data (compare with Ellithorpe et al. 1996). Thus, we initially used only the coordinates of the images of the core and of $\mathrm{G}$ in Table 2 and we excluded the flux ratios as constraints for model fitting.

We also considered object $\mathrm{X}$, which might be: (1) in the foreground of and far from $\mathrm{G}$, and therefore unrelated to the lens system; (2) at about the same redshift as G, implying that its contribution to the lensing must be estimated (note that the $R-I$ color of $\mathrm{X}$ lies between that of $\mathrm{G}$ and that of the images of the core, and therefore is marginal evidence against X sharing the redshift of G or of the QSO); and (3) in the background of G, and singly-lensed. We consider the last two possibilities below.

We assumed for most of our models that $\mathrm{G}$ is the sole contributor to the lensing in the MG0414 system (WMS95), save for including object X in one case (see below). We 
assumed that the center of brightness of $\mathrm{G}$ is also its center of mass. We used an iterative fitting algorithm (with the software suite "lensmod", see Lehár et al. 1993, modified to operate in the image plane) that treats lensed images as points instead of extended objects. The $\chi^{2}$ goodness-of-fit measure for our models is a sum of squares of position differences (each weighted by the squared inverse of its standard error) for the images of the core. We also added a similarly-weighted contribution to $\chi^{2}$ from the coordinates of $\mathrm{G}$. We found the "best-fit" lens model by direct search for minima of $\chi^{2}$ in parameter space, with the "amoeba" algorithm (see, e.g., Press et al. 1992). We analyzed in this way a series of different models $(\mathrm{A}-\mathrm{D})$, each based on a different, but simple potential.

In our first model, $\mathrm{A}$, we assumed a constant $\mathrm{M} / \mathrm{L}_{I}$ ratio for $\mathrm{G}$ (see Schneider et al. 1988), and used the elliptical isophotes for $\mathrm{G}(\S 3)$. We adjusted the $\mathrm{M} / \mathrm{L}_{I}$ ratio, represented by the radius of the Einstein ring of $\mathrm{G}$, as well as $x_{G}, y_{G}$ and the mean position angle $\Psi$ of the light distribution of $\mathrm{G}$. Thus, for model A we have 4 parameters and 8 constraints. Figure 7 shows the caustic and critical curves, and image and source positions for model A. The fit is poor, as indicated by the final $\chi^{2} / D O F$ of $\sim 13$.

For the other models, we assumed an elliptically-symmetric, two-dimensional potential $\phi$ for $\mathrm{G}$, as in Blandford \& Kochanek (1987, hereafter BK87). The adjustable parameters were $x_{G}$ and $y_{G}$; the angular core radius $\theta_{c}$; the radius, $\theta_{E}$, of the Einstein ring for $\mathrm{G}$; the ellipticity $e=1-b / a$, where $b / a$ is the axis ratio of the mass distribution, and the position angle $\Psi$ of the major axis of the mass distribution (on the sky, E of N). We did not adjust the power-law exponent $P=2-2 \alpha$ of $\phi$, where $\alpha$ is the "hardness" of BK87 (fitting experiments showed that we cannot constrain the value of $P$ usefully).

For model $\mathrm{B}$, we fixed $P=1$ and $\theta_{c}=0$, which corresponds to a singular isothermal profile; thus, we had 5 parameters and 8 constraints. Figure 7 shows the caustics and critical curves, as well as the image and source positions for model B. Compared to model A, model B yielded an improved $\chi^{2} / D O F, \sim 8$.

Model $\mathrm{C}$ was the same as model $\mathrm{B}$, except that we set $e=0$, and added an external shear parameterized by $\gamma_{e}$ as in WMS95, measured in units of the critical surface mass density $\Sigma_{c}$. Model D was the same as model C, except that we set $P=2$, which corresponds to a point mass. With both of these models, we obtained improved fits, with final $\chi^{2} / D O F$ of $\sim 5.4$ and $\sim 4.5$, respectively (see Figure 7 ). Table 4 shows the parameter values for models $B-D$. The errors quoted in the table are the changes in the parameters that separately yield an increase of $\chi^{2} / D O F$ of 1 . These errors do not account for the systematic effects that cause $\Delta \chi^{2}>1$.

The $15 \mathrm{GHz}$ flux ratios of the images of the core were recently shown to be consistent 
with no fluctuations, at the $\sim 3 \%$ rms level over a period of 6 months (Moore \& Hewitt 1996, hereafter MH96). We re-estimated the parameter values in each of our models A-D, after including the mean KH93 flux ratios as constraints, with $\sim 3 \%$ errors. We found that $\chi^{2} / D O F$ for the model A-D fits worsened by $5,12,40$ and $40 \%$, respectively. These new parameter values led to predicted image flux ratios that contributed insignificantly to $\chi^{2}$, whereas the core image coordinates contributed $2-3$ times more to $\chi^{2}$ than for the original parameter values in models A-D. Thus, we are unable to account in detail for the full set of measured properties of the images of the core.

Although we did not include them as constraints to our fits, we are able to account qualitatively for the arcs. Figure 8 shows the image and source planes for model C, with simulated source components: the four QSO images are produced by a single compact source inside the astroid caustic, and the arc is produced by an extended source that lies just inside the caustic. In VLBI observations (PP96), extended radio emission (roughly aligned with the arc emanating from B) is apparent near image B, with no counterpart near images A1 and A2. Such a configuration is consistent with the source of extended radio emission being just outside the caustic (see Figure 8).

The arc and the images of the core share geometric properties (the separation of the arc and core sources is $\sim 200 h^{-1}$ pc, according to our model $\mathrm{C}$ ), but their measured colors are dissimilar. Their mean radial distance from the center of $\mathrm{G}$ is $1.2 \pm 00^{\prime \prime} 2$, and the arc extends over a comparable distance, $\sim 1^{\prime \prime} .5$. The colors of the images of the core have a mean of $R-I=2.2 \pm 0.1 \mathrm{mag}$, and differ from the mean $R-I=1.3 \pm 0.3 \mathrm{mag}$ color for the arc. Thus, if the arc and the core sources had the same color, the measured color differences would argue in favor of reddening in the host galaxy of the QSO, instead of in the lens galaxy. However, it is possible, for example, that the arc is a distorted view of an HII region with no physical association with the QSO core, and with a bluer color. If the lens galaxy $\mathrm{G}$ were at $z=0.5$, the "most likely" value estimated by Kochanek (1992), then extinction in $\mathrm{G}$ could account qualitatively for the optical color differences among A1, A2, $\mathrm{B}$ and $\mathrm{C}$, as well as for the IR magnitudes and for the unusual line ratios found in this system (LEJT95). The color differences would arise from extinction in G that is sufficiently patchy to yield such differences on a length scale of $\sim 0^{\prime \prime} 4$, the smallest separation between the images of the core. We find no variation in the color of the arc as a function of position along it, but only at a modest $\sim 0.3$ mag level of significance, larger than the color differences among A1, A2, B, and C (Table 2). If a lack of variation were confirmed, say, at the $\sim 0.03 \mathrm{mag}$ level, it would become a much stronger argument against patchy extinction in $\mathrm{G}$, and in favor of extinction in the host galaxy of the QSO, rather than in G. Further high-resolution IR studies of the system will provide improved constraints on the location of the extinction. With B. McLeod, we intend to continue optical and IR studies both from 
the ground and with HST.

Because it appears aligned with the continuation of the arc past $\mathrm{B}$, object $\mathrm{X}$ seems morphologically associated with $\mathrm{MG}$ 0414. If it were not in the foreground of $\mathrm{G}$ (the least interesting possibility), we could have: (1) $z_{\mathrm{X}} \sim z_{\mathrm{QSO}}$, (2) $z_{\mathrm{G}}<z_{\mathrm{X}}<z_{\mathrm{QSO}}$, or $(3) z_{\mathrm{X}} \sim z_{\mathrm{G}}$. In the first case, $\mathrm{X}$ could be expected to have a counter-companion (a counter-arc in our example, or at least a second image likely to be at least as bright as $\mathrm{X}$ ) that we did not detect, and that could not have been missed by our observations (see Figure 8, dotted contour). In the second case, the redshift of $\mathrm{X}$ is not likely to exceed that of $\mathrm{G}$ significantly, because the Einstein ring radius $\theta_{E}(\mathrm{X})$ for $\mathrm{X}$ depends strongly on $z_{\mathrm{X}}$. We can illustrate this property by assuming that the mass distribution of $\mathrm{G}$ is a singular isothermal sphere, an approximation that preserves the characteristic angular scale of the lens system. For such a distribution, the radius of the multiple-image region for a source at $z_{\mathrm{X}}$ is $2 \theta_{E}(\mathrm{X})$. Within this approximation, for $\mathrm{X}$ to be a single image, $\theta_{E}(\mathrm{X})<\theta(\mathrm{X}) / 2$, where $\theta(\mathrm{X}) \sim 1^{\prime \prime}$. 5 is the angular distance from $\mathrm{X}$ to the center of brightness of $\mathrm{G}$ (Tables 1 and 2 ). However, from Table $4, \theta_{E} \sim 1^{\prime \prime} .2 \approx \theta(\mathrm{X})$; which is approximately equivalent to $\theta_{E}(\mathrm{X}) \lesssim \theta_{E} / 2$. Thus, if $z_{\mathrm{G}} \approx 1$, then $z_{\mathrm{X}}-z_{\mathrm{G}} \approx 0.4$, with this difference being smaller for any other $z_{\mathrm{G}}$. In the third case, we assumed that the $\mathrm{M} / \mathrm{L}_{I}$ ratio of $\mathrm{X}$ is equal to that of $\mathrm{G}$, and fitted a modified model $\mathrm{A}$ that included $\mathrm{X}$ in this fashion. We found $\chi^{2} / D O F \sim 41$ for this model, a very significantly worse fit than for our other models. Because $\mathrm{X}$ is clearly not point-like, we infer that it may be a dwarf galaxy near the redshift of $\mathrm{G}$, or in its foreground.

\section{Conclusions}

We conclude first that lensing is compellingly confirmed for MG 0414, in spite of the lack of a measured redshift for the lens galaxy. It seems very probable that the arc that we discovered is a highly magnified and distorted image of a patch of the source near its core. Together with the morphology of the images of the core, as refined by our observations, the arc is strong evidence in favor of lensing.

Our observations yielded positions for all 4 known images of the QSO central core, with standard errors of $0{ }^{\prime \prime} 02$, and for the lens galaxy $\mathrm{G}$ with standard errors of 0 ' 05 ; our $R$ and $I$ photometry for these objects has standard errors $\sim 0.01 \mathrm{mag}$. The photometry of the arc is much less accurate $(\sim 0.3 \mathrm{mag}$ standard errors), because it is extremely faint, especially in the $R$ filter.

The brightness distribution of $\mathrm{G}$ is well represented by a De Vaucouleurs radial profile

with effective radius $r_{e}=1^{\prime \prime} .5 \pm 0.7$ and surface brightnesss $I_{e}=24.9 \pm 0.4 \mathrm{mag} / \mathrm{arcsec}^{2}$, and 
by elliptical isophotes with ellipticity $e_{G}=0.20 \pm 0.02$ and position angle $\Phi_{G}=71^{\circ} \pm 5^{\circ}$. Thus, we conclude that $\mathrm{G}$ is most likely an elliptical galaxy, whose redshift unfortunately remains unknown.

Our lens models qualitatively account for both the compact QSO images and the arc. However, we could not find any models that were simultaneously consistent in detail with the HST positions of the images of the core and with their (unvarying, and unaffected by microlensing) radio flux ratios, as given by KH93. Monitoring of the images of MG0414 at radio frequencies shows variations at $15 \mathrm{GHz}$ of only $\leq 3.5 \%$ on time scales of 6 months (MH96). Thus, the discrepancy between observed and predicted flux ratios exhibits a serious deficiency of our models.

There are significant differences between the color of the images of the core QSO source, $R_{\text {core }}-I_{\text {core }}=2.2 \pm 0.1 \mathrm{mag}$, and that of the arc, $R_{\text {arc }}-I_{\text {arc }}=1.3 \pm 0.3 \mathrm{mag}$. The latter color appears to be invariant along the length of the arc between images A2 and $\mathrm{B}$, but at a modest level of significance. If such invariance were confirmed with high significance, we might be able to usefully test detailed extinction models. Reddening in G would likely be patchy at the same level as the observed variations among the images of the core (RMS $\sim 0.13 \mathrm{mag}$ ) over the length of the arc, and should affect the latter, as well as the former. Therefore, if our measurements were confirmed with greater accuracy, they would suggest that (at least) the bulk of the reddening occurs in the host galaxy of the QSO, rather than in G. The large discrepancies between the $R(2.50 \pm 0.04), I(2.11 \pm 0.01)$ and radio $(1.13 \pm 0.03)$ flux ratios $\mathrm{A} 1 / \mathrm{A} 2$ would then imply that microlensing is important on at least one of the paths from the core to images A1 and A2, as suggested in WMS95. Optical/IR monitoring observations could reveal microlensing, but it is possible that A2, for instance, is and would remain in a "low" state for a period of years; disentangling the possibilities could then require a very long temporal baseline.

In each of our models B-D, the estimated ellipticity of the mass distribution (or the amount of shear) is large, about twice that of the light from G. The ellipticities of the critical curves straddled by the images of the core in Figure 7 are indicative of the discordant ellipticities of the light and matter in G. The position angles of our mass models are misaligned by only $\sim 6^{\circ}$ (within our standard errors) with the orientation of the light distribution of G. Such ellipticity discrepancies are common for models of quadruple lens systems (Kochanek 1996a). Possible explanations are external shears due to, e.g., nearby objects, or local effects, such as triaxiality (Kochanek 1996b, private communication). "Reasonable" modifications of the profile of the mass distribution of G are not expected to eliminate such discrepancies.

Determination of the redshift of the lens galaxy $G$ is a very important missing 
ingredient for further analysis of MG 0414. Such an estimate could be used to derive useful physical properties of the lens and of the lensed QSO, such as masses and linear sizes. For example, for models $\mathrm{C}$ and $\mathrm{D}$, given the errors in the estimates of the positions of the core images, the mass of $\mathrm{G}$ within a radius of $\sim 1^{\prime \prime} .2$ is $M_{G}=2.1 \pm 0.1 \times 10^{11} M_{\odot}$, for $z_{G}=0.5$. Also, with the redshift of $\mathrm{G}$ measured and combined with estimates of other model parameters, one could infer useful limits for the linear sizes of the core and the arc source (WMS95), and for their separation on the sky.

The nature of object $\mathrm{X}$ is not yet determined. Its extended structure suggests that it is a galaxy. Because it is singly imaged, $\mathrm{X}$ is unlikely to be at the redshift of the QSO. If it were at the lens redshift or closer, it could not be accounted for with lens models that assume the same $\mathrm{M} / \mathrm{L}_{I}$ ratio as for model $\mathrm{A}$. However, were $\mathrm{X}$ at a redshift intermediate between the lens and the QSO, it could be a major deflector component. Difficult even for Keck-class telescopes, but very useful, would be a measurement of the redshift of X.

We gratefully acknowledge the support of ST grant G5505 and NSF grant AST93-03527. We thank C. Kochanek, B. McLeod, P. Schechter and J. Huchra for useful comments and discussions. We also thank the anonymous referee for careful, helpful suggestions.

\section{REFERENCES}

Angonin-Willaime, M.-C., Vanderriest, C., Hammer, F. \& Magain, P. 1994, A\&A 281, 388 (AVHM94)

Blandford, R. D. \& Kochanek, C. S. 1987, ApJ 321, 658 (BK87)

van Dokkum, P. G. \& Franx, M. 1996, MNRAS in press

Ellithorpe, J. D., Kochanek, C. S. \& Hewitt, J. N. 1996, ApJ in press

Falco, E. E. 1993, in Proc. 31st Liège International Colloq., ed. J. Surdej et al. (Liège: Univ. Liège), 127

Falco, E. E., Lehár, J., Wambsganss, J., Perley, R. \& Gorenstein, M. V. 1996, AJ 112, 897

Hewitt, J., Turner, E. L., Lawrence, C. R., Schneider, D. P. \& Brody, J. P. 1992, AJ 104, 968 (HTLSB92)

Hewitt, J., Burke, B. F., Turner, E. L., Schneider, D. P., Lawrence, C. R., Langston, G. \& Brody, J. P. 1989, in Gravitational Lenses, Lecture Notes in Physics, Vol. 390, ed. J. M. Moran, J. N. Hewitt, and K.-Y. Lo (Springer, Berlin) 
Holtzman, J. A., Burrows, C. J., Casertano, S., Hester, J. J., Trauger, J. T., Watson, A. M., \& Worthey, G. 1996, PASP in press

Katz, C. A. \& Hewitt, J. N. 1993, ApJ 409, 9 (KH93)

Kochanek, C. S. 1992, ApJ 384, 1

Kochanek, C. S. 1996b, preprint

Krist, J. 1996, Tiny Tim User's Manual, version 4.0, WWW STScI document, http://stsci.edu/

Lawrence, C. R., Elston, R., Jannuzi, B. T., \& Turner, E. L. 1995a, AJ 110, 2583 (LEJT95)

Lawrence, C. R., Cohen, J. G., \& Oke, J. B. 1995b, AJ 110, 2570

Lawrence, C. R. 1996, in Proc. of the 173rd Symposium of the IAU, ed. C. S. Kochanek \& J. N. Hewitt (Dordrecht: Kluwer), 299

Lehár, J., Langston, G. I., Silber, A. D., Lawrence, C. R., \& Burke, B. F. 1993, AJ 105, 847

Moore, C. \& Hewitt, J. N. 1996, submitted to ApJ (MH96)

Patnaik, A. R. \& Porcas, R. W. 1996, in Proc. of the 173rd Symposium of the IAU, ed. C. S. Kochanek \& J. N. Hewitt (Dordrecht: Kluwer), 305 (PP96)

Press, W. H., Teukolsky, S. A., Vetterling, W. T. \& Flannery B. P. 1992, in Numerical Recipes in C Second Edition (Cambridge: Cambridge U. Press)

Schechter, P. \& Moore, C. 1993, AJ 105, 1 (SM93)

Schneider, P., Ehlers, J. \& Falco, E. E. 1992, in Gravitational Lenses (New York: Springer Verlag)

Schneider, D. P., Turner, E. L., Gunn, J. E., Hewitt, J. N., Schmidt, M. \& Lawrence, C. R. 1988, AJ 96, 1755

Witt, H. J., Mao S., \& Schechter, P. 1995, ApJ 443, 18 (WMS95)

\section{FIGURE CAPTIONS}


Figure 1: PC1 5".8-field centered on MG 0414; obtained with the F675W filter $(R)$. To account for photon statistics, we used gain $g=7.12 \mathrm{e}^{-} /$count and read-out noise $r=5.24 \mathrm{e}^{-}$ for our individual exposures. We converted from pixel to equatorial coordinates $\alpha$ and $\delta$ for equinox J2000 with the IRAF STSDAS package. The software corrects for geometric distortions of WFPC2, and uses the appropriate transformations to sky coordinates from parameters incorporated in the image headers. The orientation and scale of the field on the sky are indicated by the arrows. The images of the core, object $\mathrm{X}$ and the lens galaxy $\mathrm{G}$ are all labeled.

Figure 2: PC1 23"'3-field surrounding MG0414; obtained with the F675W filter. The orientation and scale of the field on the sky are indicated by the arrows. A satellite trail is still visible, having crossed one of our exposures from $\mathrm{W}$ to $\mathrm{E}$.

Figure 3: PC1 5."8-field centered on MG 0414; obtained with the F814W filter $(I)$. The orientation and scale of the field on the sky are as in Figure 1. The emission connecting A2 and B appears fainter than in Figure 1, because the arc is bluer than the images of the core.

Figure 4: PC1 23".3-field surrounding MG0414; obtained with the F814W filter. The orientation and scale of the field on the sky are as in Figure 2.

Figure 5: Contour plot of the MEM-deconvolved $R$ image. Note that this plot is rotated relative to the orientation of Figure 1; it is aligned with the equatorial coordinate axes.

Figure 6: Circles with error bars are the derived radial profile of $\mathrm{G}$, as a function of $r^{1 / 4}$, where $r$ is the semi-major axis in arcsec. The long (short) dashes are the "best-fit" De Vaucouleurs profile to the measured profile, before (after) this profile is convolved with the PSF radial profile.

Figure 7: Caustics (solid) and critical curves (dots) for models A-D. The positions of the images of the core on the image plane are indicated with + signs. Solid ellipses are the core image position errors, traced to the source plane for each model. The goodness-of-fit of each model is indicated by the closeness to each other of these ellipses; they would all coincide in position for a perfect fit. Their sizes in each model are inversely proportional to the magnification of each core image. 
Figure 8: Caustic (top) and critical curve (bottom) for model C. Objects labeled Q, A, J and $\mathrm{X}$ on the source plane (top) correspond to similarly labeled objects on the image plane (bottom). Source Q and its images (dark) are unresolved, and correspond to the QSO core and its images. Sources A (shaded) and J (solid circles and ellipses) are resolved, and correspond to the optical arc (our observations) and the radio jets (PP96), respectively. Source X (dashed, empty circle) would be multiply imaged as object X and the elongated dashed arc (bottom), if it were at $z \sim 2.6$. 


\section{TABLE CAPTIONS}

Table 1: Right ascension and declination offsets from $\mathrm{C}$, and $R$ magnitudes of the components of MG 0414, including object X, 3 stars, and a possible group of galaxies. The coordinates of the group of galaxies are the means of the coordinates of the 3 galaxies visible in our exposures. The standard error for each coordinate is $\sigma_{\mathrm{x}, \mathrm{y}}$.

Table 2: As Table 1, for $I$ band properties, with the addition of $R-I$ colors.

Table 3: Flux ratios of the images of the core in $R$ and $I$. The combination $\mathrm{A} 1+\mathrm{A} 2$ was used to allow comparison with the ground-based estimates of SM93 and AVHM94 (see also LEJT95, Table 1).

Table 4: Model parameter values, standard errors, and $\chi^{2} / D O F$ for MG 0414. 
Table 1

\begin{tabular}{llcccc}
\hline Object & $\begin{array}{c}\Delta \alpha_{\mathrm{J} 2000} \\
(\operatorname{arcsec})\end{array}$ & $\begin{array}{c}\Delta \delta_{\mathrm{J} 2000} \\
(\operatorname{arcsec})\end{array}$ & $\begin{array}{c}\sigma_{\mathrm{x}, \mathrm{y}} \\
(\operatorname{arcsec})\end{array}$ & $\begin{array}{c}R \\
(\mathrm{mag})\end{array}$ & $\begin{array}{c}\sigma_{R} \\
(\mathrm{mag})\end{array}$ \\
\hline A1 & 1.92 & -0.30 & 0.02 & 22.760 & 0.008 \\
$\mathrm{~A} 2$ & 2.05 & 0.09 & 0.02 & 23.756 & 0.016 \\
$\mathrm{~B}$ & 1.32 & 1.63 & 0.02 & 23.488 & 0.012 \\
$\mathrm{C}$ & 0. & 0. & 0.02 & 24.258 & 0.022 \\
$\mathrm{G}$ & 0.90 & 0.32 & 0.05 & 23.227 & 0.046 \\
X & 0.46 & 1.84 & 0.05 & $>26.268$ & 0.063 \\
Star 1 & 5.89 & 10.77 & 0.02 & 21.887 & 0.004 \\
Star 2 & -0.33 & -16.92 & 0.02 & 22.997 & 0.009 \\
Star 3 & 17.59 & -13.40 & 0.02 & 23.97 & 0.02 \\
Group & -3.30 & -2.87 & 0.08 & 24.14 & 0.11 \\
\hline
\end{tabular}


Table 2

\begin{tabular}{llclclr}
\hline Object & $\begin{array}{c}\Delta \alpha_{\mathrm{J} 2000} \\
(\operatorname{arcsec})\end{array}$ & $\begin{array}{c}\Delta \delta_{\mathrm{J} 2000} \\
(\operatorname{arcsec})\end{array}$ & $\begin{array}{c}\sigma_{\mathrm{x}, \mathrm{y}} \\
(\operatorname{arcsec})\end{array}$ & $\begin{array}{c}I \\
(\mathrm{mag})\end{array}$ & $\begin{array}{c}\sigma_{I} \\
(\mathrm{mag})\end{array}$ & $R-I$ \\
\hline $\mathrm{A} 1$ & 1.92 & -0.29 & 0.02 & 20.595 & 0.003 & 2.17 \\
$\mathrm{~A} 2$ & 2.05 & 0.10 & 0.02 & 21.407 & 0.004 & 2.35 \\
$\mathrm{~B}$ & 1.32 & 1.64 & 0.02 & 21.363 & 0.004 & 2.13 \\
$\mathrm{C}$ & 0. & 0. & 0.02 & 22.212 & 0.007 & 2.05 \\
$\mathrm{G}$ & 0.86 & 0.36 & 0.05 & 21.296 & 0.014 & 1.93 \\
$\mathrm{X}$ & 0.46 & 1.84 & 0.05 & 24.769 & 0.063 & $>1.50$ \\
Star 1 & 5.88 & 10.78 & 0.02 & 21.271 & 0.004 & 0.62 \\
Star 2 & -0.33 & -16.91 & 0.02 & 22.195 & 0.007 & 0.80 \\
Star 3 & 17.58 & -13.39 & 0.02 & 22.43 & 0.008 & 1.54 \\
Group & -3.27 & -2.67 & 0.06 & 23.16 & 0.09 & 0.98 \\
\hline
\end{tabular}


Table 3

\begin{tabular}{ccc} 
Ratio & $R$ & $I$ \\
\hline $\mathrm{A} 1 / \mathrm{A} 2$ & $2.50 \pm 0.04$ & $2.11 \pm 0.01$ \\
$(\mathrm{~A} 1+\mathrm{A} 2) / \mathrm{B}$ & $2.72 \pm 0.06$ & $2.98 \pm 0.07$ \\
$\mathrm{C} / \mathrm{B}$ & $0.49 \pm 0.01$ & $0.46 \pm 0.01$ \\
\hline
\end{tabular}


Table 4

\begin{tabular}{|c|c|c|c|c|c|c|c|}
\hline Model & $\begin{array}{c}x_{G} \\
(\operatorname{arcsec}) \\
\end{array}$ & $\begin{array}{c}y_{G} \\
(\operatorname{arcsec})\end{array}$ & $\begin{array}{c}\theta_{E} \\
(\operatorname{arcsec}) \\
\end{array}$ & $\gamma_{e}$ & $e$ & $\begin{array}{c}\Psi \\
\left({ }^{\circ} \mathrm{E} \text { of } \mathrm{N}\right)\end{array}$ & $\chi^{2} / D O F$ \\
\hline B & $0.81 \pm 0.04$ & $0.31 \pm 0.02$ & $1.17 \pm 0.01$ & & $0.37 \pm 0.04$ & $78.3 \pm 0.5$ & 8.0 \\
\hline $\mathrm{C}$ & $0.83 \pm 0.04$ & $0.31 \pm 0.02$ & $1.18 \pm 0.01$ & $0.12 \pm 0.01$ & & $77.5 \pm 0.5$ & 5.5 \\
\hline D & $0.84 \pm 0.03$ & $0.30 \pm 0.02$ & $1.16 \pm 0.01$ & $0.25 \pm 0.03$ & & $77.4 \pm 0.5$ & 4.5 \\
\hline
\end{tabular}




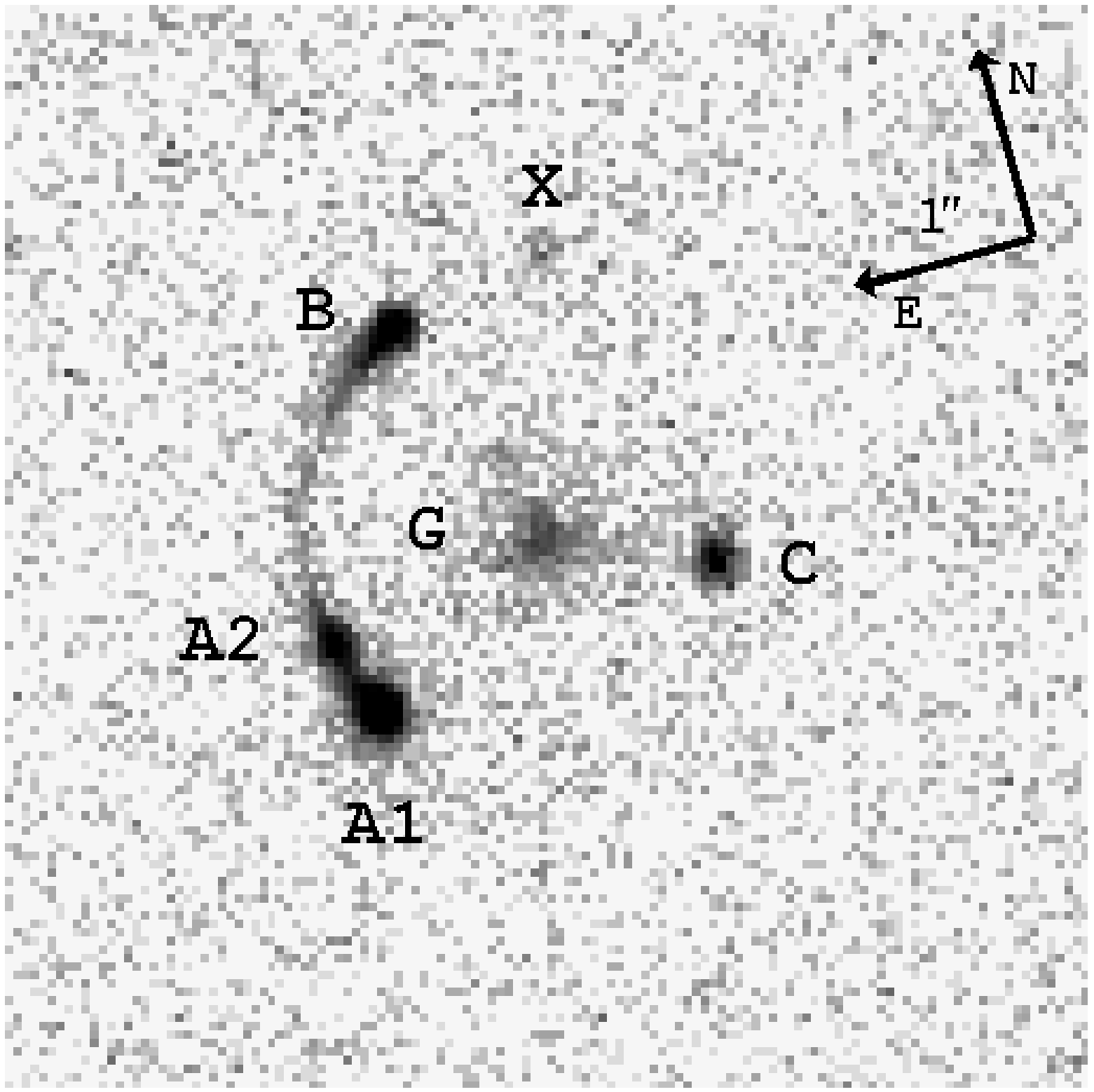




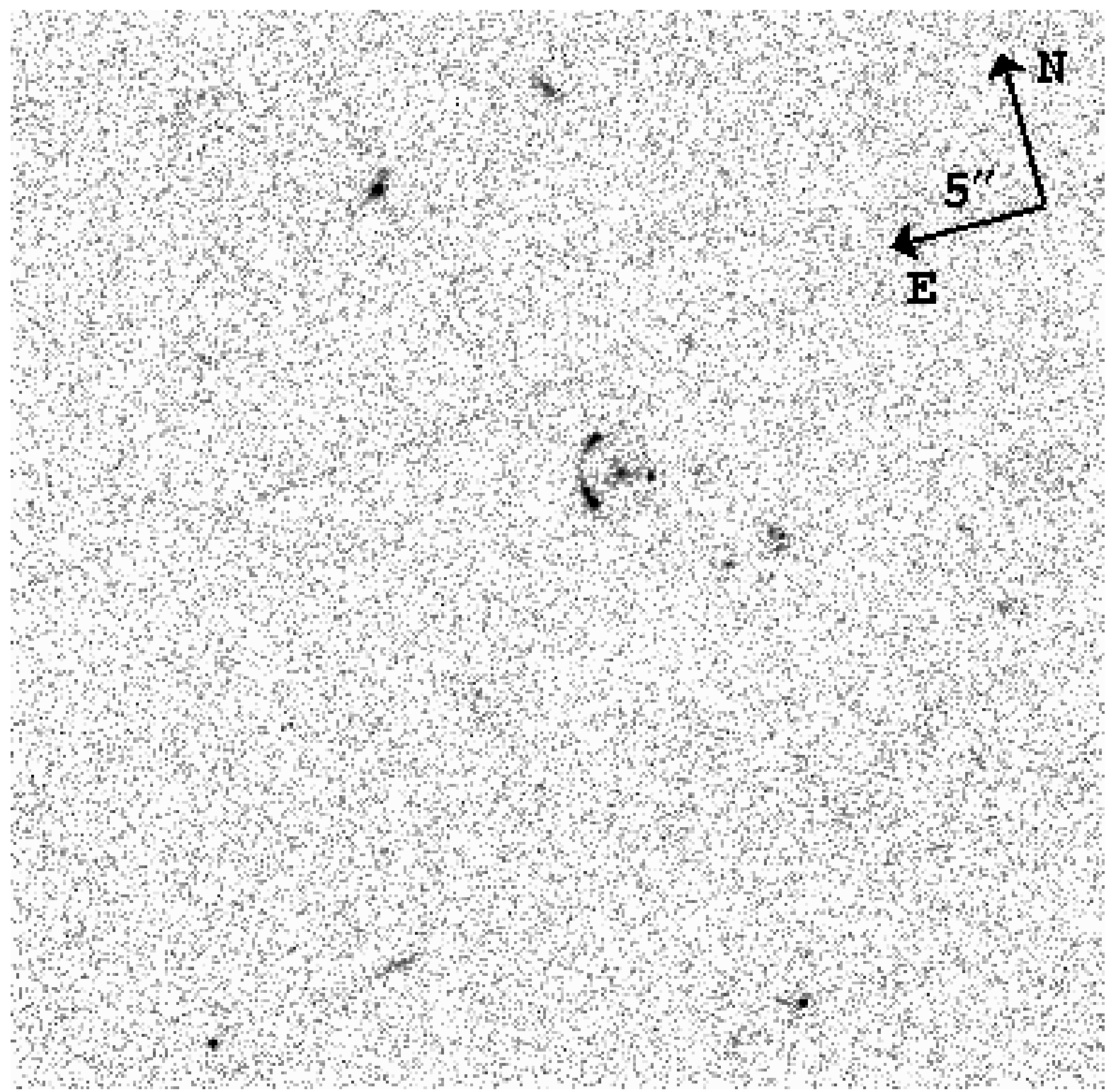




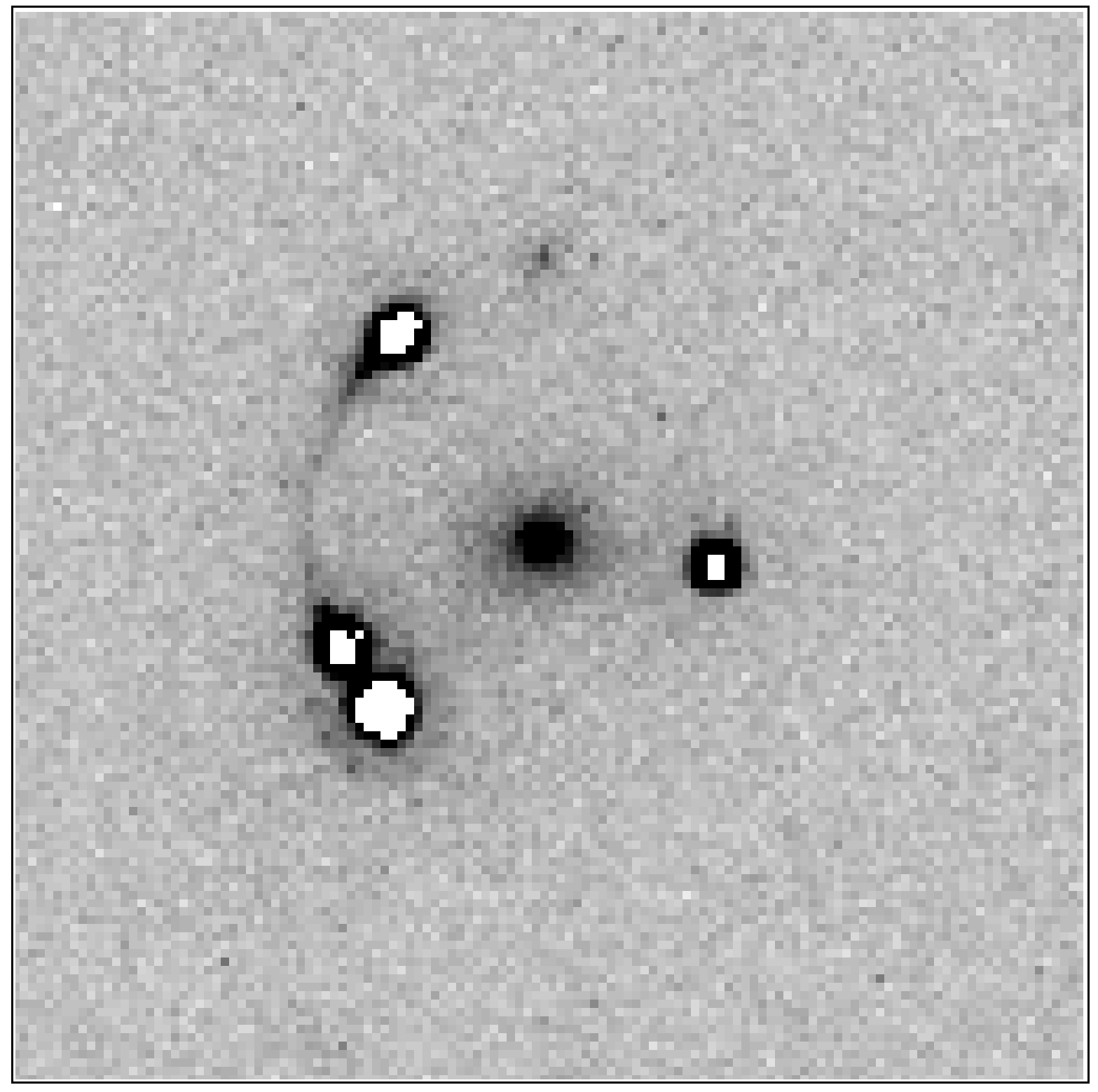




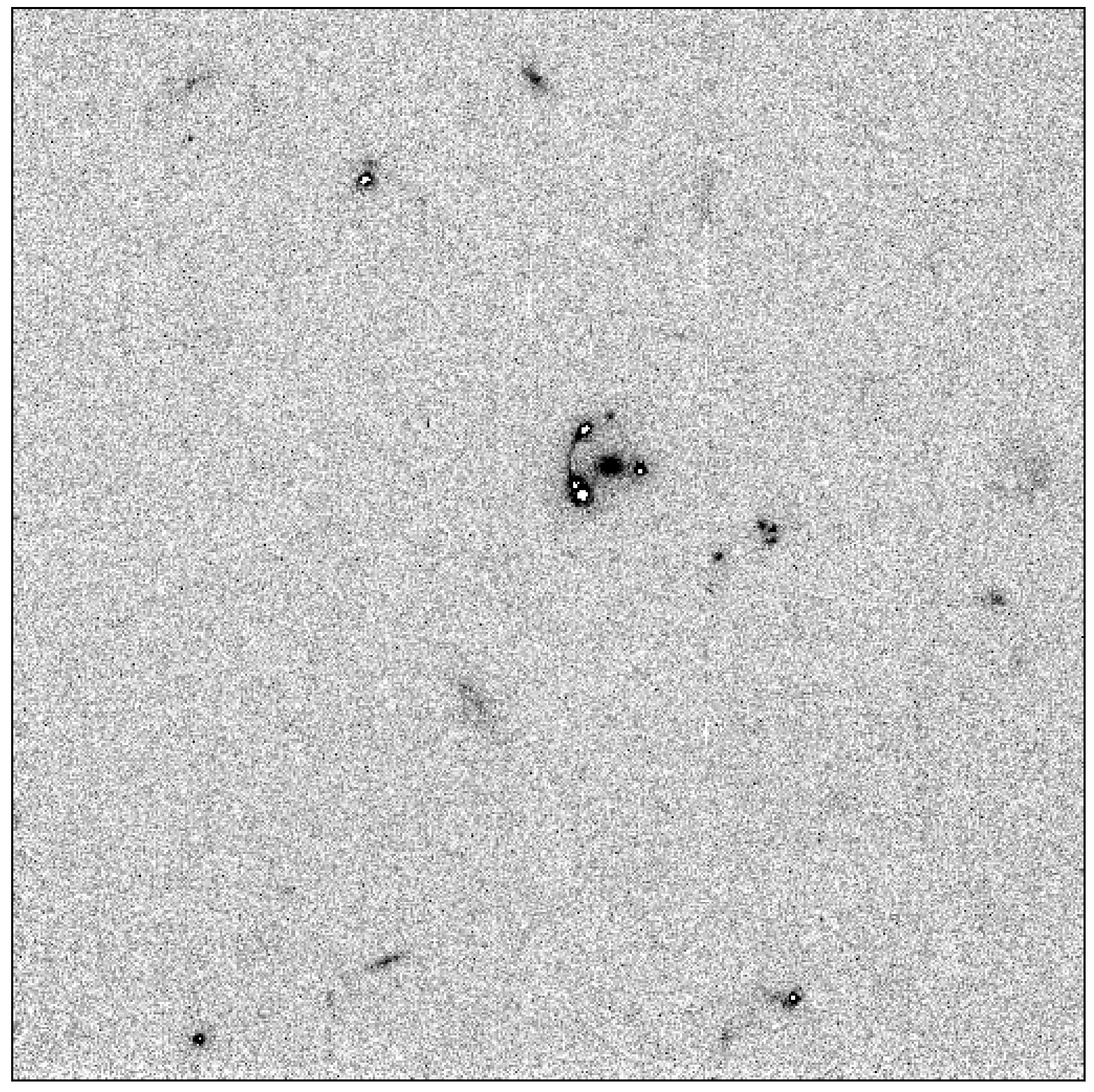




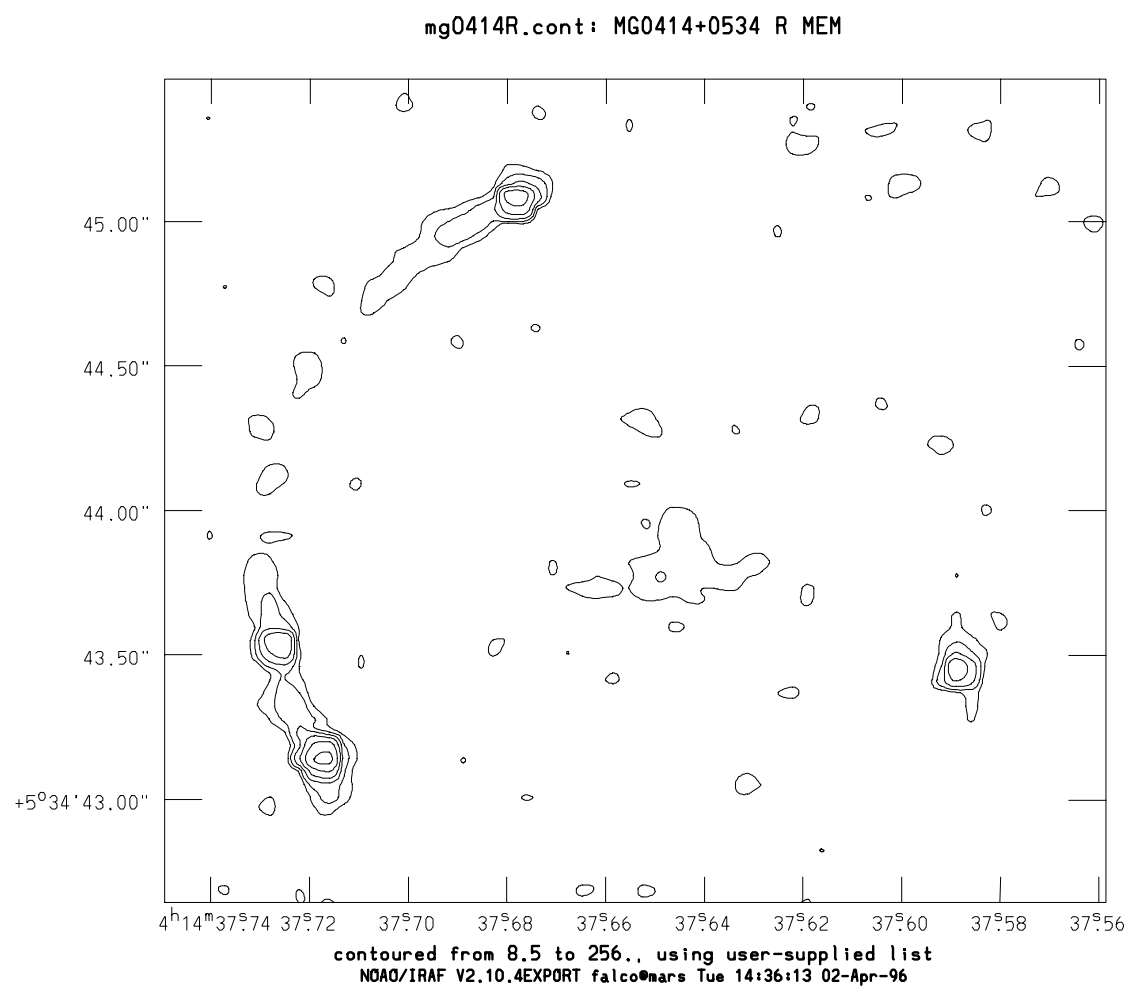




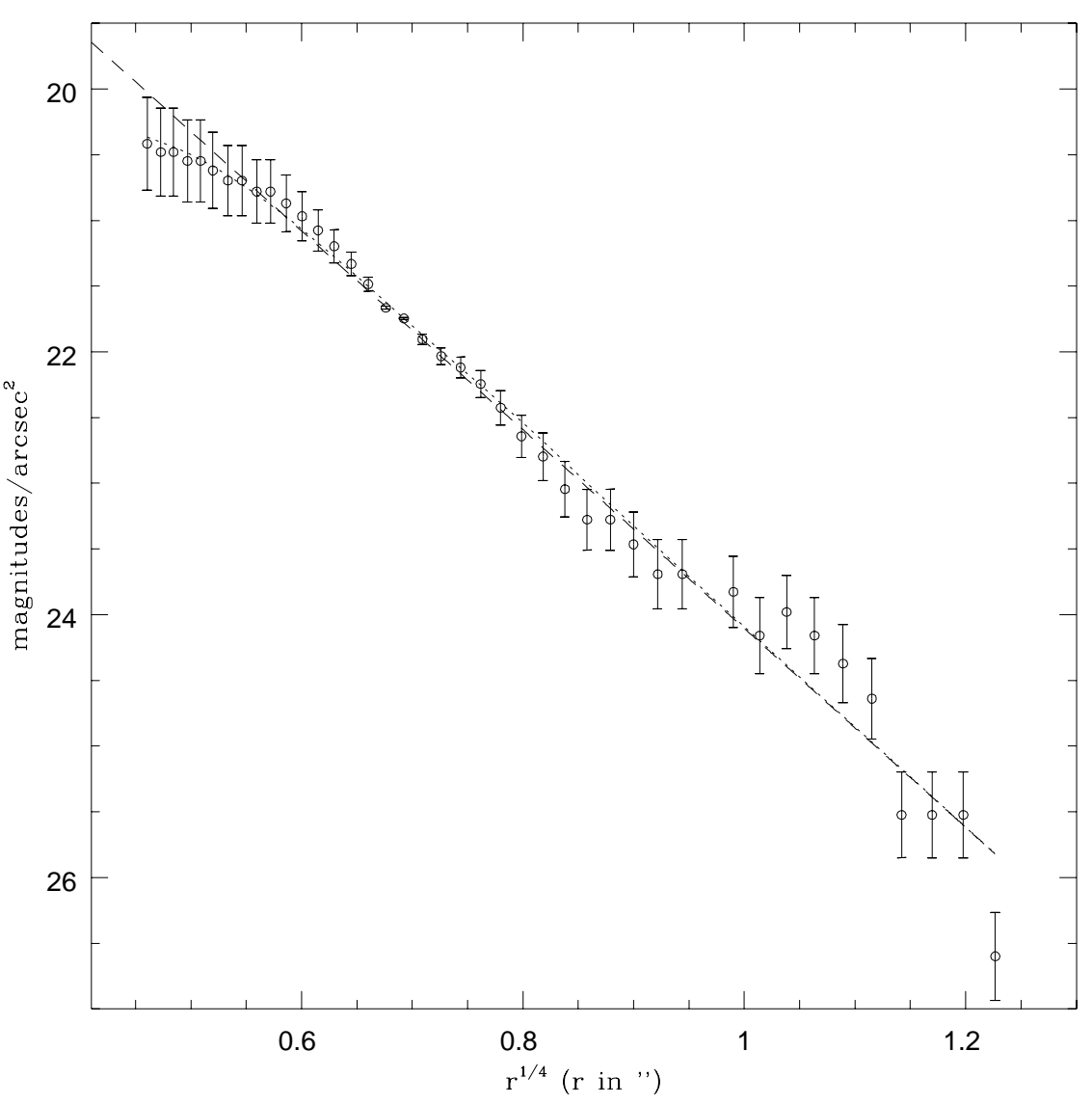




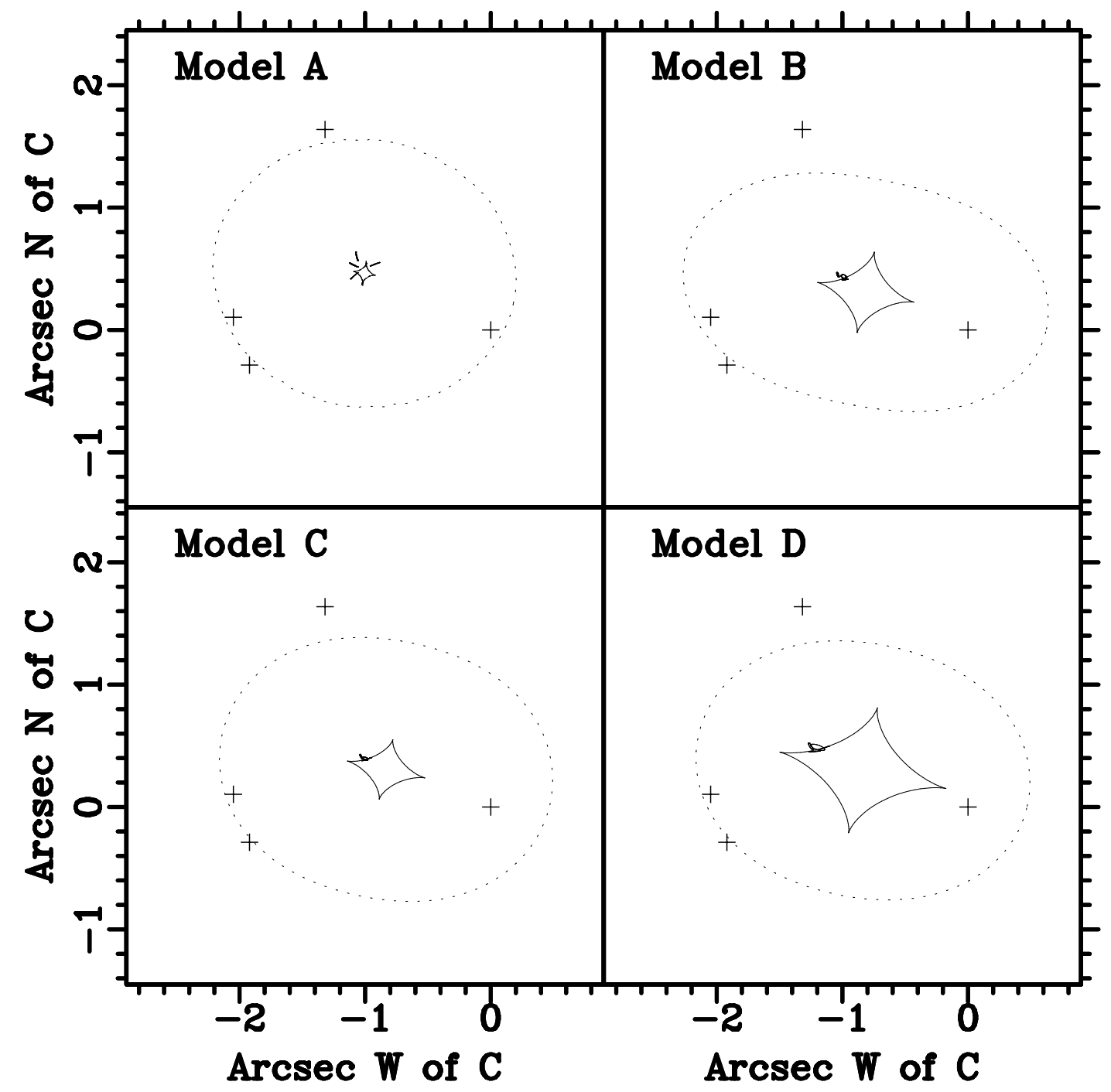



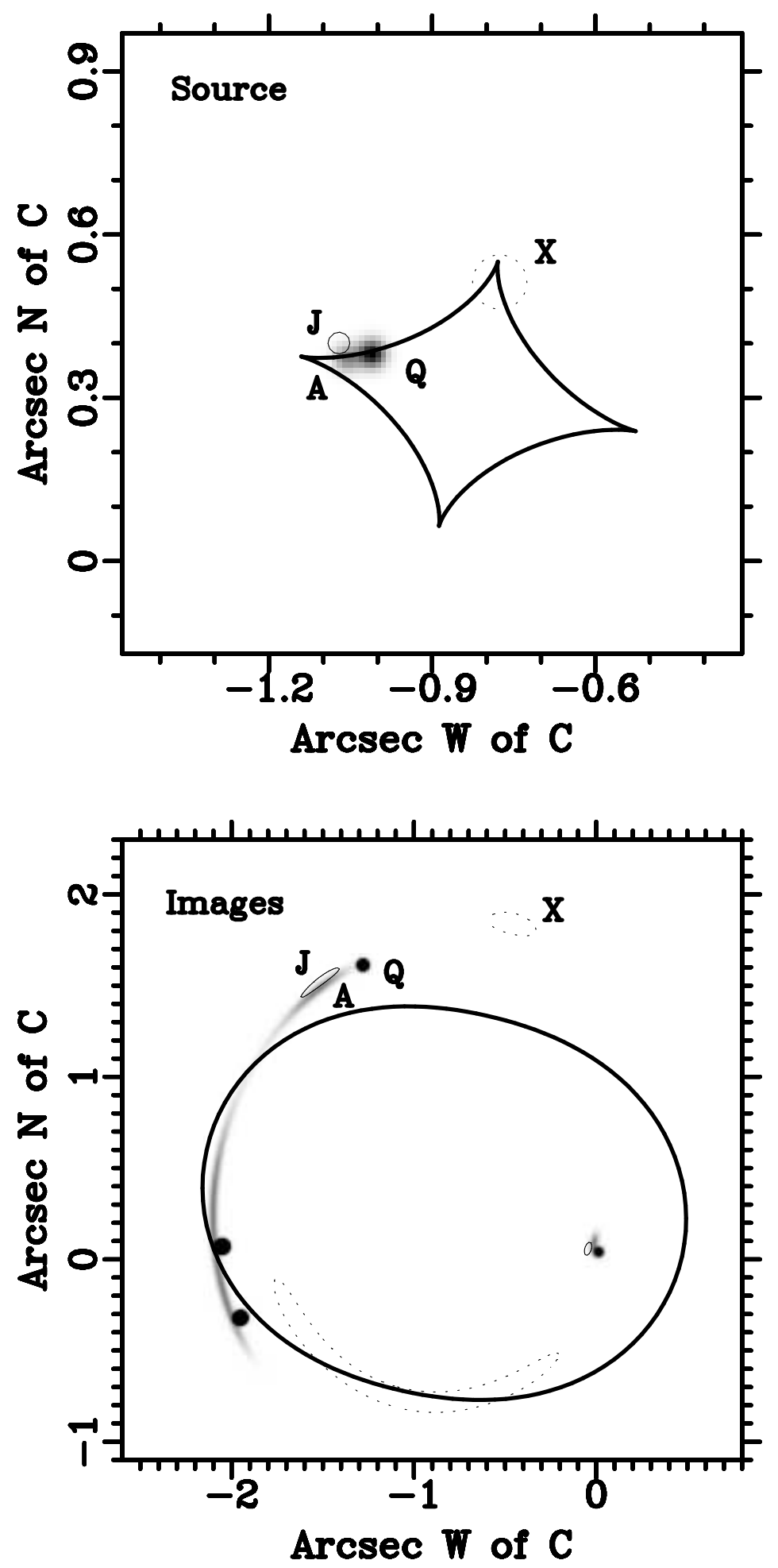